\title{
Design and Recruitment in the United States of a Multicenter Quantitative Angiographic Trial of Pravastatin to Limit Atherosclerosis in the Coronary Arteries (PLAC I)
}

\author{
Bertram Pitt, MD, Stephen G. Ellis, MD, G. B. John Mancini, MD, Howard S. Rosman, MD, and \\ Mark E. McGovern, MD, for the PLAC I Investigators
}

\begin{abstract}
The present study was designed to test the effect of pravastatin, a new, 3hydroxy-3-methylglutaryl coenzyme A reductase inhibitor, on the progression of coronary artery disease in patients with moderate hypercholesterolemia. Angiographic entry criteria included the presence of $\geq 1$ stenosis $\geq \mathbf{5 0} \%$ in a major epicardial coronary artery, and certification of film quality through the core angography laboratory. Patients qualified for randomization if after diet stabilization their low density lipoprotein cholesterol concentrations were $\geq 130$ and $<190 \mathrm{mg} / \mathrm{dl}$, and triglycerides were $\leq \mathbf{3 5 0}$ $\mathrm{mg} / \mathrm{dll}$. Pravastatin (40 $\mathrm{mg}$ ) or placebo is administered once daily at bedtime. Arteriography will be repeated at the end of 3 years of treatment. The primary end point of the study is the change in absolute mean coronary artery diameter. During a 30-month recruitment period, 44,145 patients were screened, and 408 were randomized. The most frequent reason for excluding patients during the screening and dietary lead-in periods was a low senum cholesterol level. A large proportion of patients with documented coronary artery disease have cholesterol concentrations that are considered to be normal or only modestly increased. Adherence to strict standards of quality control for digital analysis of angiograms ensures that baseline angiograms can be interpreted at the end of 3-year follow-up.
\end{abstract}

(Am J Cardiol 1993;72:31-35)

From the University of Michigan Hospital, Ann Arbor, Michigan; The Cleveland Clinic Foundation, Cleveland, Ohio; University Hospital, University of British Columbia, Vancouver, British Columbia, Canada; Henry Ford Hospital, Detroit, Michigan; and Bristol-Myers Squibb, Pharmaceutical Research Institute, Princeton, New Jersey. This study was sponsored by Bristol-Myers Squibh Company, Princeton, New Jersey. Manuscript received September 8, 1992; revised manuscript received and accepted January 20, 1993.

Address for reprints: Bertram Pitt, MD, University of Michigan Hospital, 1500 East Medical Center Drive, TC-3910-0366, Ann Arbor, Michigan 48109-0366.
$\mathrm{P}$ avastatin Limitation of Atherosclerosis in the Coronary Arteries (PLAC I) is a multicenter, prospective, randomized, placebo-controlled trial of the effect of lipid-altering therapy on the progression of coronary artery disease. The study was designed with 2 goals: The first was to evaluate the effects of monotherapy with pravastatin, a hydrophilic 3-hydroxy-3-methylglutaryl coenzyme A reductase inhibitor, on progression and regression of coronary artery disease measured by quantitative angiography. The second goal was to evaluate progression and regression of atherosclerosis in patients with only moderate increases of low-density lipoprotein (LDL) cholesterol, typical of those with symptomatic coronary artery disease. This article describes the protocol design and recruiting experience.

\section{METHODS}

The protocol was approved by the institutional review board at each study site. All patients gave written, informed consent to participate in the trial. The study was initiated in early 1988, and randomization ended after a 30-month recruitment period. All follow-up angiography will be completed in the spring of 1993.

Screening angiography: At each site (see Appendix), all patients undergoing clinically indicated coronary arteriography were screened for eligibility. The protocol for angiography required $\geq 2$ orthogonal or nearorthogonal views of all coronary segments, central placement of the catheter $(>5 \mathrm{Fr})$ in the radiographic image, administration of nitroglycerin before injection of contrast medium (in the absence of contraindications), and detailed documentation of the sequence of injections during the procedure, including the angles of view.

A patient had to have $\geq 1$ stenosis $\geq 50 \%$ in a major epicardial coronary artery to qualify for the study. The protocol required review of the documentation of the procedure, presence of an adequate portion of the angiographic catheter in a central portion of the image, digitization of eligible angiographic segments, and exclusion of excessive contrast streaming. A previously described and validated quantitative coronary artery evaluation method was used to analyze all focal stenoses in any study segment. ${ }^{1}$

Dietary stabilization/qualification for randomiza tion: Patients who fulfilled the initial screening criteria were referred to a dietician for instruction in a fat-restricted, low-cholesterol diet (American Heart Associa- 


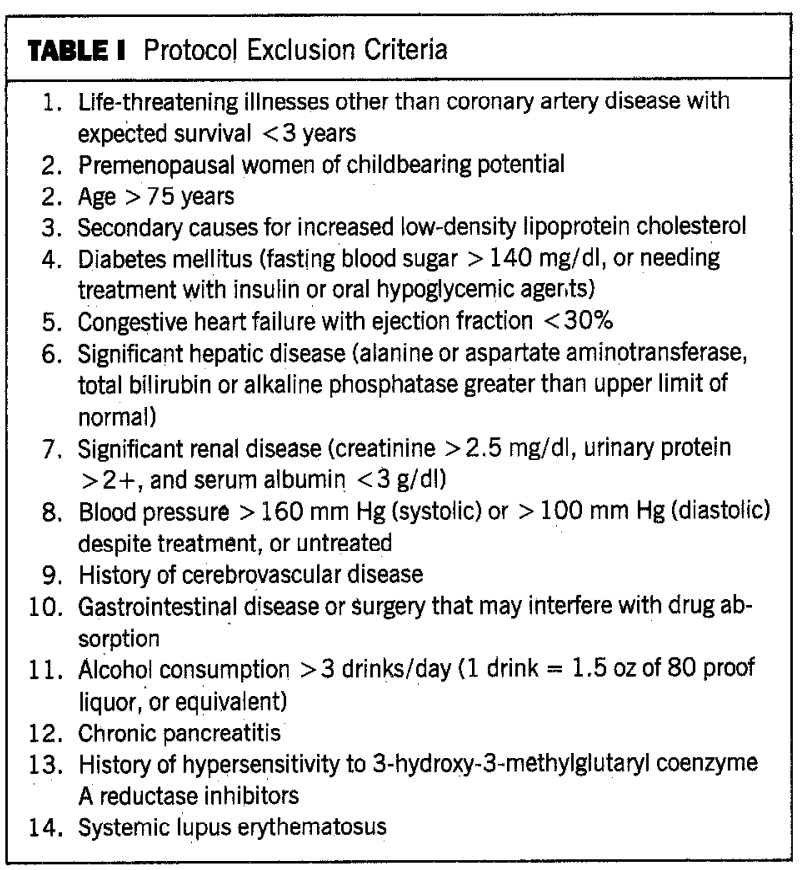

tion Phase I or equivalent ${ }^{2}$ ). After $\geq 4$ weeks of a stable diet, the first lipid profile was determined; subsequent determinations were obtained at 1- to 4-week intervals. To qualify for randomization, the average of 2 consecutive LDL cholesterol concentrations needed to be $\geq 130$ $\mathrm{mg} / \mathrm{dl}$ (3.36 mmol/liter) but $<190 \mathrm{mg} / \mathrm{dl}(4.91 \mathrm{mmol} /$ liter), and the average triglycerides $\leq 350 \mathrm{mg} / \mathrm{dl}(3.95$ mmol/liter).

Other inclusion/exclusion criteria: Before enrollment in the study, patients had to consent to coronary arteriography again at the end of 3 years of treatment. Patients receiving treatment with lipid-lowering therapy could be enrolled if an appropriate drug washout period preceded the first qualifying lipid determination. Other exclusion criteria are listed in Table I.

Dung therapy and administration: Pravastatin or matching placebo (provided by the Bristol-Myers Squibb Pharmaceutical Research Institute) is administered as two $20 \mathrm{mg}$ tablets at bedtime ( $40 \mathrm{mg}$ total), and dosage remains fixed for the duration of the study unless safety considerations dictate a reduction. Assignment to placebo or active drug is balanced within strata defined by clinical characteristics (myocardial infarction, percutaneous transluminal coronary angioplasty and stable or unstable angina) and baseline LDL cholesterol concentration (130 to 169 and 170 to $189 \mathrm{mg} / \mathrm{dl})$.

Over the course of a 3-year study, LDL cholesterol may be expected to increase to $\geq 190 \mathrm{mg} / \mathrm{dl}$, approximately the 90th percentile for the United States population in some patients. Because of the demonstrated benefits of treating patients with such marked cholesterol increase,${ }^{3}$ care was taken to identify and treat them. If the mean of 3 consecutive LDL cholesterol levels is $\geq 190 \mathrm{mg} / \mathrm{dl}$ in any patient, personnel at the site are to be notified in writing to advance the diet to the American Heart Association Phase II or equivalent diet. ${ }^{2}$ To maintain study integrity and blinding, a patient from the other treatment group matched by age, sex and upper tertile of LDL cholesterol concentration is to be chosen to change diet also. If LDL cholesterol does not decrease to $<190 \mathrm{mg} / \mathrm{dl}$ after 1 month, the patient and matching subject are to be instructed to begin taking cholestyramine resin beginning with 1 packet (bid) titrated to a maximum of 6 packets/day. Patients whose levels remain $\geq 190 \mathrm{mg} / \mathrm{dl}$ despite addition of cholestyramine are to be administered 5 to $10 \mathrm{mg}$ of open-label pravastatin (or placebo, depending on original treatment assignment). If these measures are unsuccessful, the patient is withdrawn from the study.

Evaluation of patients: Patients are to return to the clinic every 6 weeks for the first 18 months of the study for safety evaluation (liver-enzyme elevation and adverse events), and every 12 weeks thereafter. Follow-up arteriography is to be performed after 36 months, or earlier, if feasible, in the event of withdrawal from the study, or coronary artery bypass graft surgery. As each patient completes the study, the paired baseline and follow-up cineangiograms (blinded to order and patient identification) will be reviewed by an experienced angiographer who will select matching views for analysis. (The preliminary core laboratory reading will be used only for qualification of the film.)

Interim monitoring/policy atvisony boand: The conduct of the study is reviewed semiannually by a policy advisory board of 5 members (see Appendix) who are independent of the sponsor and trial organization.

Statistical considerations/end point analysis plans: Original sample-size calculations were based on the limited data then available on progression rates and variability from the Coronary Artery Surgery Study. ${ }^{4}$ A total sample size of 400 subjects was deemed adequate to demonstrate a $40 \%$ treatment effect. More recent quantitative data enable more accurate sample-size estimates. ${ }^{5}$ Assuming a decrease in mean coronary artery diameter of $0.20 \mathrm{~mm}$ in the placebo group, an SD of $0.34 \mathrm{~mm}, 80 \%$ power and 0.05 significance level (2-sided), the detectable treatment effect ranges between 51 and $55 \%$ for 350 and 300 patients, respectively, completing the study (allowing for withdrawals).

The primary end point of the study is the rate of progression for mean diameter averaged over the following 10 segments: left main, proximal and mid-left anterior descending, largest diagonal, proximal and distal left circumflex, largest obtuse marginal, and proximal, midand distal right. Any angiogram obtained $\geq 90$ days after initiation of double-blind therapy can be included in the analysis of the primary end point. The primary analysis will be by intention-to-treat. Rates of progression will by analyzed using rank-based tests (e.g., rank-sum tests) and expressed as a slope $(\mathrm{mm} / \mathrm{month})$. (This results in the variable being weighted for time on trial.) If a final angiogram is not available in a subject because of death or myocardial infarction, a value will be imputed for that patient equal to the greatest progression rate observed in the group completing 36 months in the trial, corrected for duration of follow-up. Arteries and segments that undergo angioplasty or coronary artery bypass graft will be excluded from the study. (Patients undergoing coronary artery surgery during the study will be defined as having reached a study end point, and the angiogram before surgery will be included in the analysis.) 
Several secondary angiographic and lipoprotein end point analyses are planned (Table II). Secondary clinical end points include occurrence of myocardial infarction, stroke, angioplasty and coronary artery bypass graft surgcry, and total and cause-specific mortality.

\section{RESULTS}

Recruitment began in early 1988 and ended approximately 2.5 years later (Figure 1). Tables III and IV list the reasons why patients were excluded during the screening and dietary lead-in periods. The most frequent reason for excluding a patient during the early screening phase was low serum cholesterol level. In general, subjects were enrolled in the dietary stabilization phase if the screening total cholesterol level was $\geq 210$ to 220 $\mathrm{mg} / \mathrm{dl}$, although there was considerable variation from site to site, and the threshold depended on whether a fatrestricted diet was already being followed. Based on the accumulating experience, several changes were made in the protocol and conduct of the study (including increas-

\begin{tabular}{|l|}
\hline TABLE II Secondary End Points \\
\hline Angiographic measurements \\
Mean minimal diameter \\
Mean maximal diameter \\
Mean percent stenosis \\
Development of new lesions \\
Change in average severity of lesions \\
Average no. of lesions and new lesions/pt. \\
Lesion changes stratified by baseline severity \\
Incidence of progression, regression and mixed progression/regression, \\
or no change \\
Lipoprotein and apolipoproteins \\
Total and low-density lipoprotein cholesterol, high-density lipoprotcin \\
cholesterol, high-density lipoprotein2, and high-density lipoprotein 3 \\
Triglycerides \\
Apolipoproteins A-1 and B \\
Lipoprotein (a)
\end{tabular}

ing the number of participating centers) to enable randomization to be completed successfully. The protocol revision having the greatest impact on enrollment was changing the lower limit required for mean LDL cholesterol from 150 to $130 \mathrm{mg} / \mathrm{dl}$ (Figure 1). This finding provides additional support for the qualitative observation that most patients with coronary artery disease have only moderate increases of total and LDL cholesterol.

Other revisions with only modest effects on recruitment included increasing the upper age limit from 70 to 75 years, and the upper limit for triglycerides from 300 to $350 \mathrm{mg} / \mathrm{dl}$, and allowing subjects with stable or un-

\begin{tabular}{|lc|}
\hline TABLE III Patients Excluded After Screening \\
\hline Patients screened & 44,145 \\
Patients excluded & $43,031^{*}$ \\
Screening total cholesterol too low & 11,006 \\
Too ill & 5,068 \\
Norrial curoniary arteries & 4,509 \\
Diabetes mellitus & 3,975 \\
Age & 3,968 \\
Concomitant illnesses or prohibited medications & 3,862 \\
Angiography protocol not followed & 3,134 \\
No qualifying clinical or angiographic criteria & 1,138 \\
Refused to participate & 1,104 \\
Other/not specified & 5,267 \\
\hline *|ncludes 1,155 patients receiving lipid-lowering drugs. \\
\hline
\end{tabular}

TABLE IV Reasons for Disqualification in Patients Enrolled But Not Randomized

$\begin{array}{lr}\text { Patients enrolled } & 1,114 \\ \text { Patients excluded } & 706 \\ \text { Low density lipoprotein cholesterol concentration too low } & 240 \\ \text { Triglyceride concentration too high } & 25 \\ \text { Patient withdrew } & 166 \\ \text { Angiogram rejected } & 54 \\ \text { Other/not specified } & 221\end{array}$

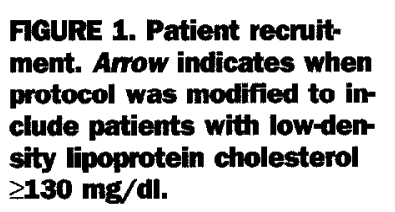

FGGURE 1. Patient recruitment. Arrow indicates when protocol was modified to include patients with low-den$\geq 130 \mathrm{mg} / \mathrm{dll}$.

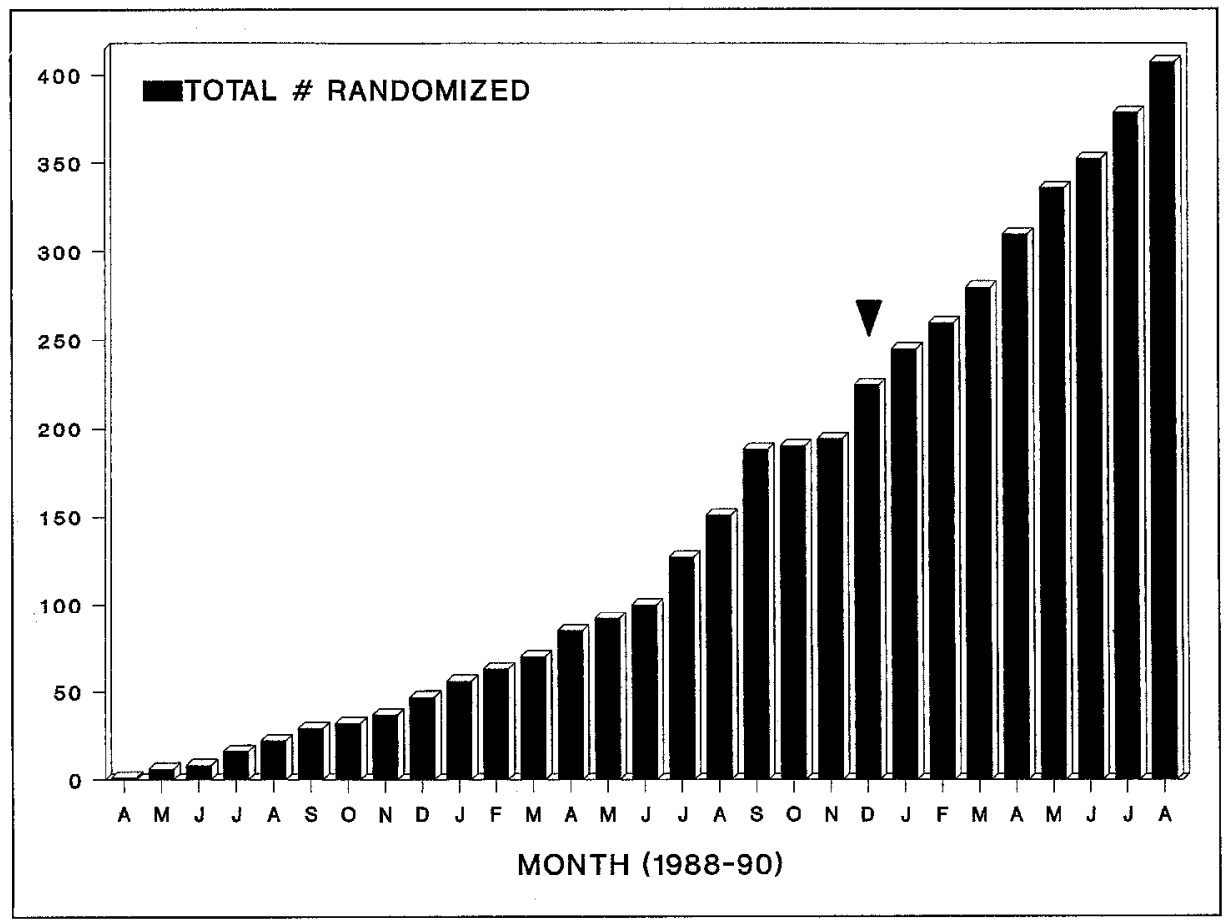


TABLE V Reasons for Rejection of Baseline Angiograms by Core Angiography Laboratory

Excessive streaming
Excessive editing
No qualifying lesion
Absent or inadequate views
3-vessel bypass
Nitroglycerin not given
Inadequate documentation
Automated catheter tracking not possible
Angioplasty urgently needed
Total films rejected
Total films screened

stable angina (in addition to those after myocardial infarction or angioplasty) to participate in the study.

Tables III and IV show that some patients were excluded from the study because either the angiographic protocol was not followed, or qualifying clinical or angiographic criteria were absent, or both. Some patients were included whose angiograms were sent for certification, but were not accepted after analysis in the core angiography laboratory. Table $\mathrm{V}$ shows the reasons for rejecting these films. The overall acceptance rate was $90.1 \%$ of submitted films. In contrast, in the early phases of the study, the acceptance rate was as low as $71 \%$. Protocol adherence and film quality improved after the reasons for rejection were reviewed with the clinical sites.

Baseline demographic characteristics of the 408 patients randomized are listed in Table VI.

\section{DISCUSSION}

PLAC I and other ongoing studies should help establish whether treatment of patients with moderately increased serum cholesterol levels slows progression of coronary artery disease and presumably prevents clinical events as much as in trials of those with severe hypcrcholcstcrolcmia. ${ }^{6,7}$ The positive or negative results should have important implications for the large number of patients with coronary artery disease but only moderate LDL cholesterol elevation.

Several features of the design and recruitment of patients in the trial deserve comment, and may bear on the design of future studies. Assessment of the progression of coronary artery disease was the primary aim. Thus, before randomization, each patient's angiogram was screened through the core angiography laboratory to ensure that the protocol was followed and that the angiogram could be sufficiently digitized to ensure adequate analysis at the end of 3-year follow-up. In the early phases of the study, $>100$ angiographers were participating, and variation in attention to contrast streaming and protocol adherence was considerable. After core laboratory personnel rejected several films, protocol adherence improved substantially. Table $\mathrm{V}$ documents the value of using this approach in conjunction with quantitative angiography to ensure adequacy of baseline films.

The most frequent reason for excluding patients from the study was low serum cholesterol (Table III). A large proportion of patients with documented coronary artery disease have cholesterol concentrations only modestly

\begin{tabular}{|c|c|c|}
\hline & Group A & Group B \\
\hline Mean age (years) & 57 & 57 \\
\hline Range & $37-75$ & $33-72$ \\
\hline Men/women (\%) & $76 / 24$ & $79 / 21$ \\
\hline \multicolumn{3}{|l|}{ Race $(\%)$} \\
\hline White & 90 & 86 \\
\hline Other & 10 & 14 \\
\hline Average total cholesterol $(\mathrm{mg} / \mathrm{dl})$ & 229 & 232 \\
\hline Range $^{\star}$ & $172-294$ & $175-294$ \\
\hline $\begin{array}{l}\text { Average low-density lipoprotein cholesterol } \\
(\mathrm{mg} / \mathrm{dl})\end{array}$ & 162 & 165 \\
\hline Range* & $119-220$ & $113-208$ \\
\hline $\begin{array}{l}\text { Average high-density lipoprotein cholesterol } \\
(\mathrm{mg} / \mathrm{dl})\end{array}$ & 41 & 41 \\
\hline Range* & $23-98$ & $25-70$ \\
\hline Average triglycerides $(\mathrm{mg} / \mathrm{dl})$ & 165 & 167 \\
\hline Range* & $49-511$ & $46-425$ \\
\hline
\end{tabular}

increased or within the range considered normal for the U.S. population. The data support conclusions from other studies ${ }^{8,9}$ and have important implications not only for future trials but also for understanding the pathogenesis of coronary artery disease. Mechanisms other than hyperlipidemia, such as insulin resistance, may be of substantial pathophysiologic importance in patients with only moderate or no increase in serum LDL cholesterol. Although a lipid-lowering strategy such as monotherapy with pravastatin is likely to benefit patients with severe or moderately severe hypercholesterolemia, the effect of reducing lipids in those with only minimal or no increase in serum lipids despite coronary artery disease should be determined.

\section{APPENDIX}

Study organization: PARTICIPATING INSTTTUTIONS AND PRINCIPAL INVESTIGATORS: University of Michigan Hospital, Ann Arbor, MI: Bertram Pitt, MD, Stephen G. Ellis, MD; Henry Ford Hospital, Detroit, MI: Howard S. Rosman, MD; Sinai Hospital, Detroit, MI: Melvyn Rubenfire, MD; William Beaumont Hospital, Royal Oak, MI: Gerald C. Timmis, MD; Saint Joseph Hospital, Ann Arbor, MI: Ronald Van den Belt, MD; Deaconess Hospital, St. Louis, MO: Harold L. Kennedy, MD; Georgetown University Medical Center, Washington, D.C.: Charles E. Rackley, MD; Burns Clinical Research Foundation, Petoskey, MI: Harry T. Colfer, MD; Johns Hopkins University, Baltimore, MD: Lewis C. Becker, MD; Humana Heart Institute, Louisville, KY: David A. Dageforde, MD; Central Ohio Interventionists, Columbus, OH: Richard J. Candela, MD; New Mexico Heart Clinic, Albuquerque, NM: George F Leatherman, MD; Washington Adventist Hospital, Tacoma Park, MD: Robert DiBianco, MD; and Core Angiography Laboratory University Hospital, University of British Columbia, Vancouver, British Columbia, Canada: G. B. John Mancini, MD, Director.

POLICY ADVISORY BOARD: W. Virgil Brown, MD, Emory University, Atlanta, GA; Harold T. Dodge, MD, University of Washington, Seattle, WA; Richard A. Kron$\mathrm{mal}, \mathrm{PhD}$, University of Washington, Seattle, WA; Albert Oberman, MD, University of Alabama, Birming- 
ham, $A L$; and Jeffrey Raines, $\mathrm{PhD}$, University of Miami, Miami, FL.

CENTRAL LIPID ANALYTIC LABORATORY: Lipid Research Center, Washington University School of Medicine, St. Louis, MO: Thomas G. Cole, $\mathrm{PhD}$, Director.

CENTRAL APOLIPOPROTEIN ANALYTIC LABORATORY:

Center for Cardiovascular Research, Sinai Hospital of

Detroit, Detroit, MI: James J. Maciejko, PhD, Director.

1. Mancini GBJ, Simon SB, McGillem MJ, LeFree MT, Friedman HZ, Vogel RA. Automated quantitative coronary arteriography: morphologic and physiologic validation in vivo of a rapid digital angiographic method. Circulation 1987,75:452-460.

2. Nutrition Committee, American Heart Association. Dietary guidelines for healthy American adults. A statement for physicians and health professionals by the Nutrition Committee, American Heart Association. Circulation 1986;74:1465A-1468A. 3. Lipid Research Clinics Program. The Lipid Research Clinics Coronary Primary Prevention Trial results. I. Reduction in incidence of coronary heart disease. IAMA $1984 ; 251: 351-364$.
4. Ellis S, Sanders W, Goulet C, Miller R, Cain KC, Lesperance J, Bourassa MG, Alderman EL. Optimal detection of the progression of coronary artery disease: comparison of methods suitable for risk factor intervention trials. Circulation 1986;74: $1235-1242$.

5. Watts GF, Lewis B, Brunt JNH, Lewis ES, Coltart DJ, Smith LDR, Mann J, Swan AV. Effects on coronary artery disease of lipid-lowering diet, or diet plus cholestytamine, in the St. Thomas' Atherosclerosis Regression Study (STARS). Lancet 1992;339:563-569.

6. Blankenhom DH, Nessim SA, Johnson RL, Sanmarco ME, Azen SP, CashinHemphill L. Beneficial effects of combined colestipol-niacin therapy on coronary atherosclerosis and coronary venous bypass grafts. JAMA 1987;257:3233-3240

7. Brown G, Albers JJ, Fisher LD, Schaefer SM, Lin J-T, Kaplan C, Zhao X-Q, Bisson BD, Fitzpatrick VF, Dodge HT. Regression of coronary artery disease as a result of intensive lipid-lowering therapy in men with high levels of apolipoprotein B. N Engl J Med 1990;323;1289-1298.

8. Pekkanen J, Linn S, Heiss G, Suchindran CM, Leon A, Rifkin BM, Tyroler HA. Ten-year mortality from cardiovascular disease in relation to cholesterol level among men with and without preexisting cardiovascular disease. $N$ Engl J Med 1990;322: $1700-1707$.

9. Romm PA, Green CE, Reagan K, Rackley CE. Relation of serum lipoprotein cholesterol levels to presence and severity of angiographic coronary artery disease. Am J Cardiol 1991;67:479-483. 